\title{
Neonatal Nephromegaly Due to Homozygous Variant in the DIS3L2 Gene is Consistent with the Genetic Diagnosis of Perlman Syndrome
}

\author{
Al-Buali Majed J*, Al-Sunini Muna M, Al-Faraj Jaffer S, Al-Shams Ahmed A, Al-Mohammed Salah M, Zaal Hani R and Al- \\ Mousa Haider $\mathrm{H}$
}

Medical Genetic Unit, Department of Pediatrics, Maternity Children Hospital Alhassa, Hofuf, Saudi Arabia

\begin{abstract}
Background: Perlman syndrome is an uncommon genetic disorder grouped with overgrowth syndrome in which an abnormal increase in the size of the body or a body part of the infant often noticed at birth. The disorder,usually affects the kidneys as main findings. Perlman syndrome inherited as an autosomal recessive pattern. People with this condition are generally born with renal abnormalities also called renal hamartomas, nephroblastomatosis also been grouped with Renal cell carcinoma. The characteristic features include polyhydramnios, fetal overgrowth, including macrocephaly, neonatal macrosomia, visceromegaly mainly hepatomegaly and nephromegaly, dysmorphic facial features, and an increased risk for Wilms' tumor at an early age. The prognosis of Perlman syndrome is poor with high morbidity and mortality rate.
\end{abstract}

Material and Methods: We report 3 months old female infant from Saudi origin product of consanguineous marriage with prenatal sonographic signs suggestive of Perlman Syndrome specifically polyhydramnios, bilateral nephromegaly with fetal ascites. The clinical course was marked by neonatal respiratory distress, cyanosis and refractory hypoxemia with chylothorax required mechanical ventilation. Frequent hospitalization since that due to frequent attacks of apnea and chest infections.

Result: The constellation of clinical presentation and radiological finding confirmed by Molecular investigations showed a homozygous variant c.1810C $>\mathrm{T} p .\left(\mathrm{Gln} 604^{*}\right)$ in the DIS3L2 gene (OMIM : 614184 ) which is consistent with Autosomal Recessive Perlman syndrome.

Keywords: Perlman Syndrome • Bilateral Neonatal Nephromegaly $•$ Neonatal Chylothorax $•$ Polyhydramnios $•$ Fetal Ascites $•$ DIS3L2 Gene

\section{Introduction}

Perlman syndrome is an uncommon genetic disorder grouped with overgrowth syndrome in which an abnormal increase in the size of the body or a body part of the infant often noticed at birth. The disorder, usually affects the kidneys as main findings. Perlman syndrome inherited as an autosomal recessive pattern. People with this condition are generally born with renal abnormalities also called renal hamartomas, nephroblastomatosis also been grouped with Renal cell carcinoma. The characteristic features include polyhydramnios, fetal overgrowth, including macrocephaly, neonatal macrosomia, visceromegaly mainly hepatomegaly and nephromegaly, dysmorphic facial features, and an increased risk for Wilms' tumor at an early age [1-6].

The prevalence is $<1 / 1000000$. So far, around 30 patients have been reported in the literature [1]. Perlman syndrome (OMIM; 267000) is an autosomal recessive syndrome due to homozygosity or compound heterozygosity for mutations in the DIS3L2 gene. DIS3 MITOTIC CONTROL, S. CEREVISIAE, HOMOLOG-LIKE 2; DIS3L2) [2,3].

The first six patients were born to a consanguineous Yemenite Jewish family whose renal morphology was described by Liban and Kozenitsky and clinical characteristics by Perlman et al. [4,7].

Neri et al. further delineated the syndrome in two affected sibs and proposed the name Perlman syndrome [8]. Additional patients have been described since that, an about 28 patient with wide variety of signs and symptoms associated with multiple congenital anomalies and multisystemic complications have been reviewed by Alessandri et al. [9].
Affected children are large at birth, hypotonic, and show characteristic facial dysmorphisms (inverted V-shaped upper lip, prominent forehead, deepset eyes, broad and flat nasal bridge, and low-set ears), renal anomalies (nephromegaly and hydronephrosis), Perlman syndrome is associated with a high risk of Wilms tumor, with a $64 \%$ incidence in infants surviving beyond the neonatal period. Other findings that occur with Perlman syndrome are mental retardation, neurodevelopmental disorders, corpus callosum agenesis, macrosomia, visceromegaly, hepatosplenomegaly, hypoglycemia secondary to pancreatic islet cell hyperplasia, and muscular hypotonicity including the abdominal wall muscles. Isolated findings include diaphragmatic hernia, interrupted aortic arch, anomalous left coronary artery, and polysplenia can be a manifestation of Perlman syndrome and additional features of volvulus and distal ileal atresia $[3-6,8,10]$.

Prenatal diagnosis of Perlman syndrome has been reported [11]. The most frequent antenatal sonographic findings are polyhydramnios, macrosomia, nephromegaly and visceromegaly in the second and third trimester. Fetal ascites is another prenatal finding occasionally described (six cases) [11-13].

The prognosis of Perlman syndrome is poor with a high neonatal mortality rate. Most of the affected infants develop respiratory distress with refractory hypoxemia and/or renal failure and died within the first hours or days of life. Long-term abdominal distension due to enlarged kidneys and massive ascites can induce hypoplastic lungs [9,14].

Few affected patients survive the neonatal period. Most of them died during the first year due to sepsis or progressive respiratory insufficiency. Only few patients have been described with survival beyond the first year of life. All had developmental delay of variable degree. The girl reported by Piccione et al. is the unique exception, having normal neurological and cognitive development at 9 years $[9,14,15]$.

*Address for Correspondence: Dr. Al-Buali Majed J, Deputy Chairman of Medical Genetic Unite, Pediatrics Department, Maternity Children Hospital Alhassa, Hofuf, Saudi Arabia, E-mail: doctormajed1@gmail.com

Copyright: @ 2020 Al-Buali MJ, et al. This is an open-access article distributed under the terms of the Creative Commons Attribution License, which permits unrestricted use, distribution, and reproduction in any medium, provided the original author and source are credited. 
Management of Perlman syndrome syndrome is different for each individual depending on the features observed and often involves the coordinated efforts of a multidisciplinary team of specialists. Future research should focus on the clinical manifestation and, genotype-phenotyperelationships, and the development of evidence-based treatments as well as genetic counselling and prevention measures.

Here, we report a new case, an infant with prenatal findings polyhydramnios, fetal ascites as well as macrosomia. Postnatalfindings includes bilateral nephromegaly, muscular hypotonia, developmental delay in addition to frequent attacks of hypoxemia and respiratory failure.

\section{Method}

\section{Human subject}

In the present study, we clinically investigated a single affected individual (proband) from Saudi origin family. The proband underwent a comprehensive clinical evaluation by a general pediatrician, radiologist and clinical geneticist.

\section{Molecular genetic test}

Whole Exome Sequence was requested to reach a diagnosis and to rule out other possibilities of similar genetic condition conditions. Whole Exome Sequence have been done at centogene. RNA capture baits against approximately $60 \mathrm{Mb}$ of the Human Exome (targeting $>99 \%$ of regions in CCDS, RefSeq and Gencode databases) is used to enrich regions of interest

from fragmented genomic DNA with Agilent's SureSelect Human All Exon V6 kit. The generated library is sequenced on an Illumina platform to obtain an average coverage depth of $\sim 100 x$. Typically, $\sim 97 \%$ of the targeted bases are covered $>10 x$. An end to end in-house bioinformatics pipeline including base calling, alignment of reads to GRCh37/hg19 genome assembly, primary filtering out of low quality reads and probable artefacts, and subsequent annotation of variants, is applied. All disease causing variants reported in $\mathrm{HGMD} \AA$, in ClinVar or in CentoMD ${ }^{\circledR}$ as well as all variants with minor allele frequency (MAF) of less than $1 \%$ in gnomAD database are considered. Evaluation is focused on coding exons along with flanking $+/-20$ intronic bases. All pertinent inheritance patterns are considered. In addition, provided family history and clinical information are used to evaluate eventually identified variants.. All variants related to the phenotype of the patient, except benign or likely benign variants, are reported. Centogene has established stringent quality criteria and validation processes for variants detected by NGS. Lower quality single nucleotide or deletion insertion variants are thus being confirmed by Sanger. As a result of this we warrant specificity of $>99.9 \%$ for all reported variants.

\section{Result}

The DIS3L2 variant c.1810C $>\mathrm{T}$ p. $\left(\mathrm{G} \ln 604^{*}\right)$ creates a premature stop codon. It is classified as likely pathogenic (class 2) according to the recommendations of Centogene and American College of Medical Genetics and Genomics (ACMG).

\section{MCH Case Report}

We presented a female infant was the second child of healthy consanguineous Saudi parents from Eastern province. A previous pregnancy had resulted in a healthy female infant. The 28-year-old mother reported no medical problems, no exposure to teratogens or alcohol. The mother she was on regular follow antenatally because of polyhydramnios. At 36 weeks of gestation, an ultrasound examination showed macrosomia fetus with enlarged kidneys and fetal ascites.

AT 37 weeks of gestation the fetus delivered by cesarean section because of polyhydramnios and macrosomia. Apgar score was 6,8 at 1 and 5 minutes respectively. The newborn weighted $3,320 \mathrm{~g}$ (75th centile) with a length of 52 $\mathrm{cm}$ (75th centile) and a head circumference of $32.5 \mathrm{~cm}$ (50th centile). Enlarged kidneys were palpable. The newborn showed soft dysmorphism. Facial anomalies include round face, full cheeks, prominent forehead, depressed nasal bridge, epicanthal folds, deep-set eyes, everted upper lip, low set abnormally modeled ears and micrognathia.

The infant was intubated and ventilated immediately soon after birth due to frequent attacks of apnea. The thorax appeared bell-shaped associated with abdominal distension and abdominal muscular hypoplasia.

Abdominal ultrasound scan showed both kidneys are enlarged (Right kidney measured $7.18 \mathrm{~cm} \mathrm{X} 3.03 \mathrm{~cm}$ ) and (Left kidney measured $6.6 \mathrm{~cm} \mathrm{X}$ $3 \mathrm{~cm}$; for 97 th centile) with increased parenchymal echogenicity, and poor corticomedullary differentiation as well as innumerable tiny cystic changes (white arrow) (Figure 1,2). Both kidneys were normal in anatomic position without evidence of calculi or hydronephrosis, minimal ascites, normal spleen and liver. Transfontanellar cranial ultrasound scan was normal. Chest radiography suggested bilateral pleural effusion (chylothorax component was confirmed by thoracentesis). The skeleton was unremarkable on radiograph. Upper gastrointestinal study with barium showed grade 3 gastroesophageal reflex diseases. Echocardiography reported to have large Patent ductus arteriosus and Patent foramen ovals.

The clinical course was marked by respiratory distress, frequent attacks of apnea, refractory hypoxemia for which the newborn required mechanical ventilation for 21 days pleural effusion due to congenital chylothorax which required thoracentesis to improve ventilation.

The infant readmitted many times after discharge due to respiratory distress, attacks of apnea, poor feeding with frequent chest infection with frequent aspiration pneumonia in which required NGT insertion. Facial dysmorphism become more obvious specifically full face appearance with micrognathia. Developmental the infant having persistent head lag with Generalized hypotonia, abdominal muscular hypoplasia in addition to Absence of social smile or any social interaction were noticed. This infant at the age of 6 months was brought dead in the mid night and the cardiopulmonary resuscitation process was completed, but the infant died and was diagnosed as case of sudden infant death syndrome most likely duo to repeated attacks of apnea.

\section{Discussion}

The current patient is the first case to be reported from Saudi Arabia. The

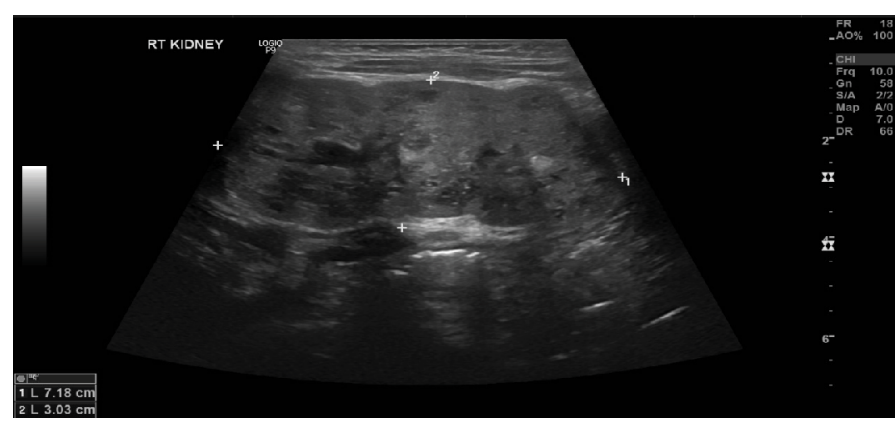

Figure 1: Bilateral nephromegaly with mild increased parenchymal echogenicity, and poor corticomedullary differentiation as well as innumerable tiny cystic changes (white arrow): RT Kidney.

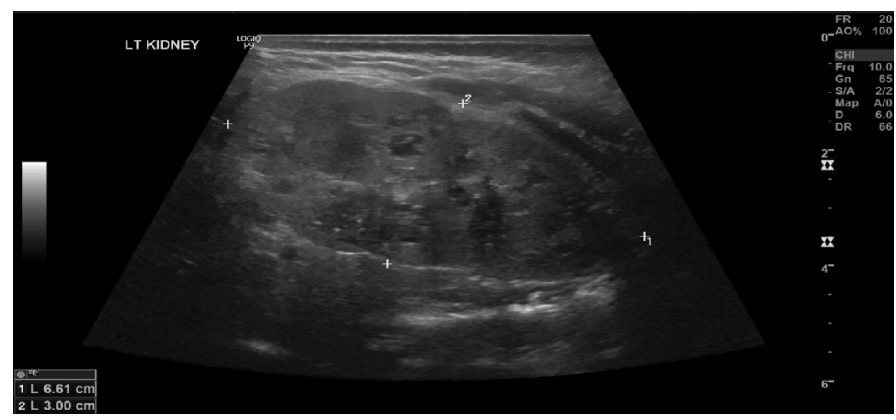

Figure 2: Bilateral nephromegaly with mild increased parenchymal echogenicity, and poor corticomedullary differentiation as well as innumerable tiny cystic changes (white arrow): LT Kidney. 
proband has the clinical presentation suggesting of Perlman syndrome since birth with main presentation bilateral nephromegaly, antenatally found to have fetal ascites, fetal macrosomia and polyhydramnios unexplained frequent attacks of hypoxemia and apnea one of the noticed clinical problems. All mention features have been reported in one or more cases reviewed by [9].

However, the current patient lacked some major features, which are macrosomia, hepatosplenomegaly and hyperplasia of the pancreas. On the other hand, dysmorphic features become obvious with time including round face, full cheeks, prominent forehead, depressed nasal bridge, epicanthal folds, deep-set eyes, everted upper lip, low set abnormally modeled ears and micrognathia .Additionally, the current patient had pleural effusion due to congenital chylothorax were never given.

Some syndromes considered as differential diagnosis for Perlman syndrome. Clinical overlap between Perlman syndrome and two other neonatal overgrowth syndromes, Beckwith-Wiedemann Syndrome (BWS) and Simpson-Golabi-Behmel Synsrome (SGBS), has been emphasized. Some patients with Perlman syndrome may have been initially diagnosed as having BWS until the results of renal histology or the occurrence of another case in the sibship $[11,16]$.

However the current patient have no macroglossia, abnormal tongue, ears creases or hemi hyperplasia which is well known findings in BWS absence of polydactyly and cleft lip and palate as well being a female make SGBS less likely.

Other differential diagnoses have been reported. Findings in Perlman syndrome such Abdominal distension, and enlarged echogenic kidneys with loss of corticomedullary differentiation led to a diagnosis of polycystic kidney (PKD) disease in two other fetal cases $[15,17]$.

The DIS3L2 gene mapped to chromosome $2 q 37.2$ based on an alignment of the DIS3L2 sequence (GenBank BC030113) with the genomic sequence (GRCh37). The Gene Function described in transfected COS-7 and HeLa cells. Astuti et al. observed that DIS3L2 was localized predominantly in the cytoplasm. RNA degradation assays using transfected HEK293 cells demonstrated that DIS3L2 has exonuclease activity. Knockdown studies in HeLa cells showed that DIS3L2 inactivation was associated with mitotic abnormalities and altered expression of mitotic checkpoint proteins, with lowered expression of TTK (604092), aurora B (AURKB; 604970), and phosphorylated CDC25C (157680), but upregulation of cyclin B1 (CCNB1; 123836), RAD21 (606462), and securin (PTTG1; 604147). DIS3L2 overexpression suppressed the growth of human cancer cell lines, and knockdown enhanced the growth of those cells. Astuti et al. concluded that DIS3L2 has a critical role in RNA metabolism and is essential for the regulation of cell growth and division [3].

The pluripotency factor LIN28 (611043) blocks the expression of LET7 (605386) microRNAs in undifferentiated cells during development by binding to pre-LET7 RNAs and recruiting RNA uridyltransferases ZCCHC11 (613692) and ZCCHC6 (613467) to uridylate pre-LET7. The identity of the RNase that degrades uridylated pre-LET7 was unknown. Chang et al. (2013) identified Dis312 as the 3-prime-5-prime exonuclease responsible for the decay of uridylated pre-let7 in mouse embryonic stem cells. Biochemical reconstitution assays showed that 3-prime oligouridylation stimulates DIS3L2 activity in vitro, and knockdown of DIS3L2 in mouse embryonic stem cells leads to the stabilization of pre-let7. Chang et al. concluded that their study established 3-prime oligouridylation as an RNA decay signal for DIS3L2 and identified the first physiologic RNA substrate of this exonuclease $[18,19]$.

The current patient has more syndromic features and multisystemic complication more what reported to for PKD. Moreover, the radiological findings not showing the characteristic cystic changes in (PKD) to reaching the definitive diagnosis in such clinical presentation and to figure our patient belong to any of these syndromes, molecular genetic test. Whole Exome Sequence have been done and the result supporting the diagnosis of Perlman syndrome. For the first time in the literature, we reported a novel mutation that has not been previously reported. (OMIM 614814)In our patient, the DIS3L2 variant c. $1810 \mathrm{C}>\mathrm{T}$ p.(Gln604*) creates a premature stop codon. It is classified as likely pathogenic (class 2) according to the recommendations of Centogene and ACMG.

\section{Conclusion}

Perlman syndrome is a rare entity syndrome and considered one of differential diagnosis for newborn with visceromegaly. We alert clinicians to consider the possibility of perlman syndrome, whenever there are constellation of prenatal findings, polyhydramnios, fetal ascites with neonatal bilateral nephromegaly. Molecular genetics test specifically Whole Exome Eequence (WES) is very helpful to reach a definitive diagnosis and to rule other differential diagnosis such as BWS, SGB as well as autosomal recessive polycystic disease. Furthermore to delineate the long term of medical care, the outcome and genetic counselling.

Finally we emphasize the need for longitudinal data; as such information will provide a profile encompassing care recommendation. Future research is needed in order to elucidate the long-term outcome of these patients.

\section{Acknowledgement}

The authors would like to acknowledge the treating team as well as the parent of the patient for their kind cooperation.

\section{Consent of Publication}

Not applicable

\section{Ethical Approval}

Ethical approval is not required at our institute to publish an anonymous case report.

\section{Funding}

None.

\section{Declaration of Conflict of Interest}

The authors declare that there is no conflict of interest.

\section{References}

1. www.orphanet.net

2. http://www.omim.org/entry/26700

3. Astuti, Dewi, Mark R Morris, Wendy N Cooper and Raymond HJ Staals, et al "Germline Mutations in DIS3L2 Cause the Perlman Syndrome of Overgrowth and Wilms Tumor Susceptibility". Nature Genet 44 (2012): 277-284.

4. Perlman, Max, GM Goldberg, J Bar-Ziv, and G Danovitch. "Renal Hamartomas and Nephroblastomatosis with Fetal Gigantism: A Familial Syndrome". J Pediat 83 (1973): 414-418.

5. Perlman, Max, M Levin, and B Wittels. "Syndrome of Fetal Gigantism, Renal Hamartomas and Nephroblastomatosis with Wilms' Tumour". Cancer 35 (1975): 1212-1217.

6. Perlman, Max, John M Opitz, and James F Reynolds. "Perlman Syndrome: Familial Renal Dysplasia with Wilms Tumor, Fetal Gigantism, and Multiple Congenital Anomalies". Am J Med Genet 25 (1986): 793-795.

7. Liban, Erich, and Isidoro L Kozenitzky. "Metanephric Hamartomas and Nephroblastomatosis in Siblings". Cancer 25 (1970): 885-888.

8. Neri, Giovanni, Maria Enrica Martini-Neri, Ben E. Katz, and John M. Opitz. “The Perlman Syndrome of Familial Renal Dysplasia with Wilms Tumor, Fetal Gigantism, and Multiple Congenital Anomalies". Am J Med Genet 19 (1984): 195-207. 
9. Alessandri, Jean-Luc, Fabrice Cuillier, Duksha Ramful and Sandrine Ernould, et al. "Perlman Syndrome: Report, Prenatal Findings and Review". Am J Med Genet 146A (2008): 2532-2537.

10. Henneveld, Hetty Th, Richard A van Lingen, Ben CJ Hamel and Irene StolteDijkstra. "Perlman Syndrome: Report of a Case and Results of Molecular Studies". Am J Hum Genet 45 (1989): A48.

11. Chitty, Lyn S, Terry Clark, and Darryl Maxwell. "Perlman Syndrome: A Cause of Enlarged, Hyperechogenic Kidneys". Prenatal Diagn 18 (1998): 1163-1168.

12. Greenberg, Frank, Fernando Stein, Mary V Gresik and Milton J Finegold, et al. "The Perlman Familial Nephroblastomatosis Syndrome”. Am J Med Genet 24 (1986): 101-110.

13. Elliott, Margaret, Rosemary Bayly, Trevor Cole and I Karen Temple. "Clinical Features and Natural History of Beckwith-Wiedemann Syndrome. Presentation of 74 New Cases". Clin Genet 46 (1998): 168-174.

14. Henneveld, Hetty Th, Richard A van Lingen, Ben CJ Hamel and Irene Stolte-
Dijkstra. “Perlman Syndrome: Four Additional Cases and Review". Am J Med Genet 86 (1999): 439-446.

15. Piccione, Maria, Massimiliano Cecconi, Mario Giuffre, Margherita Lo Curto, et al "Perlman Syndrome: Clinical Report and Nine-Year Follow-Up". Am J Med Genet 139A (2005): 131-135.

16. Verloes, Alan, Brigitte Massart, Isabelle Dehalleux and Jean-Paul Langhendries. "Clinical Overlap of Beckwith-Wiedemann, Perlman and Simpson-Golabi-Behmel Syndromes: A Diagnosis Pitfall". Clin Genet 47 (1995): 257-262.

17. Coppin, Brian, Isabella Moore, and Eli Hatchwell. "Extending the Overlap of Three Congenital Overgrowth Syndromes". Clin Genet 51 (1997): 375-378.

18. Schilke, Kathrin, Franz Schaefer, Rüdiger Waldherr and Wiltrud Rohrschneider, et al. "A Case of Perlman Syndrome: Fetal Gigantism, Renal Dysplasia, and Severe Neurological Deficits". Am J Med Genet 91 (2000): 29-33.

19. Chang, Hao-Ming, Robinson Triboulet, James E Thornton, and Richard I Gregory. "A Role for the Perlman Syndrome Exonuclease DIS3L2 in the Lin28-Let-7 Pathway". Nature 497 (2013): 244-248.

How to cite this article: AAl-Buali Majed J, Al-Sunini Muna M, Al-Faraj Jaffer $S$ and Al Shams Ahmed A, et al. "Neonatal Nephromegaly Due to Homozygous Variant in the DIS3L2 Gene is Consistent with the Genetic Diagnosis of Perlman Syndrome". J Mol Genet Med 14 (2020): 447 doi: 10.37421/jmgm.2020.14.447 